\section{Living in the past}

Phys. Rev. A 89, 024102 (2014)

Don't dwell on the past - especially when it is poorly defined, as in the case of quantum particles. Traditionally, referring to a particle between measurements has not made much sense, but in the context of delayed-choice experiments the issue cannot be ignored.

Lev Vaidman suggested that the history of a particle would be betrayed by the weak trace it leaves along its path: any interaction with the environment should leave some trace and, provided that it is weak enough not to disturb the overall evolution of the system, ensemble measurements on pre- and post-selected particles would reveal that trace. He illustrated the idea using photons travelling within a nested Mach-Zehnder interferometer: as the photons are reflected by the mirrors in the interferometer arms, their paths are exposed by the mirror vibrations.

Vaidman now provides an interpretation of these results in terms of the overlap between forward and backward evolving wavefunctions. Intriguingly, the particle can exist not only within the overlap, but also in adjacent regions - suggesting a weaker 'secondary presence' that, despite not leaving a trace of its own, would still affect the particle.

\section{Cold flow}

Astron. J. 147, 48 (2014)

Star-forming galaxies constantly need raw material, yet surveys of neutral hydrogen gas ( $\mathrm{H} \mathrm{I})$ in nearby active galaxies can only account for about $10 \%$ of the necessary accreting gas. This cold, low-density gas should flow near low-mass galaxies - but these very traits make the gas difficult to detect. And once detected, it is hard to say whether the gas is feeding a galaxy or is the result of a galactic outflow due to high starformation activity.

So far, the only sign of such a cold flow lies between the galaxies M31 and M33; close up, however, the flow looks clumpy, like clouds. Daniel Pisano set out to find more examples using the Green Bank Telescope in West Virginia, USA.

In the southern sky, NGC 2997 is a nearby spiral galaxy in a cluster of eight gas-rich galaxies. NGC 6946 is another nearby spiral galaxy with at least three gasrich neighbours. Both of them turn out to have extended low-density trails of $\mathrm{H} \mathrm{I}$, and for NCG 6946 in particular, that structure is filamentary, seeming to connect it to its companion galaxies.

\section{Tiny lights}

Phys. Rev. Lett. 112, 047403 (2014)

Gaël Reecht and colleagues have built the first single-molecule light-emitting diode, taking the latest step in device miniaturization and edging towards the realization of a molecular computer.

Reecht et al. suspended a single polythiophene molecule between the tip of a scanning tunnelling microscope and a gold substrate. They found that their device emitted light when current passed in a certain direction, with negligible effect when the polarity was reversed. The optical spectra measured by the team were insensitive to shifts in voltage, implying that the emissions corresponded to the intrinsic energy levels of the polymer itself.

Organic light-emitting diodes are already used in visual displays for mobile phones and digital cameras, offering high-quality luminescence at relatively low cost. The device reported in this study is reminiscent of these diodes, but boasts a significantly smaller scale than has previously

been achieved.

\section{Picturing paints}

Proc. Natl Acad. Sci. USA 111, 1708-1713 (2014)

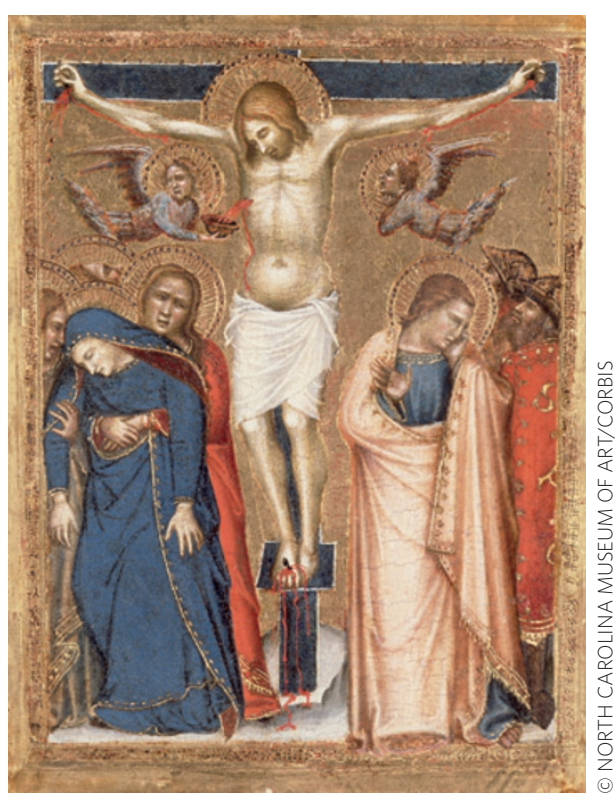

The scientific study and conservation of our cultural heritage relies a great deal on modern imaging and spectroscopic techniques, especially on non-destructive methods that alleviate the need to take samples from valuable pieces of art.

Tana Villafana and colleagues have now adapted 'femtosecond pump-probe microscopy' - a non-destructive biomedical imaging technique, typically applied in analysing skin tissue - for use in conservation science.

In pump-probe microscopy, molecules are electronically excited by a laser pulse train; their response is then probed by a second pulse train. If particular frequencies of the two pulses are chosen, the distribution of pigment molecules on the surface of a painting can be traced.

The authors applied their technique to a fourteenth-century painting, The Crucifixion (pictured) by Italian painter Puccio Capanna. Virtual crosssections of the robes of Mary and of one of the 'floating angels' reveal differing compositions of the pigments - in particular, of natural ultramarine, which is obtained from the mineral lapus lazuli and in the fourteenth century was more expensive than gold.

Written by May Chiao, Iulia Georgescu, David Gevaux, Abigail Klopper and Bart Verberck 